\title{
ARTICLE
}

\section{Extremal problems for GCDs}

\author{
Ben Green ${ }^{1}$ and Aled Walker ${ }^{2 *}$ \\ ${ }^{1}$ Mathematical Institute, University of Oxford, Andrew Wiles Building, Radcliffe Observatory Quarter (550), Woodstock \\ Road, Oxford, OX2 6GG, UK and ${ }^{2}$ Trinity College, Trinity Street, Cambridge, CB2 1TQ, UK \\ *Corresponding author. Email: aw530@cam.ac.uk
}

(Received 16 December 2020; revised 12 March 2021; first published online 8 April 2021)

\begin{abstract}
We prove that if $A \subseteq[X, 2 X]$ and $B \subseteq[Y, 2 Y]$ are sets of integers such that $\operatorname{gcd}(a, b) \geqslant D$ for at least $\delta|A||B|$ pairs $(a, b) \in A \times B$ then $|A||B| \ll_{\varepsilon} \delta^{-2-\varepsilon} X Y / D^{2}$. This is a new result even when $\delta=1$. The proof uses ideas of Koukoulopoulos and Maynard and some additional combinatorial arguments.
\end{abstract}

2020 MSC Codes: Primary 11A05; Secondary 11B75

\section{Introduction and proof strategy}

Fix $\varepsilon \in(0,1)$ throughout the paper; implied constants and thresholds may depend on $\varepsilon$ but are otherwise absolute. Let $p_{0}$ be a threshold which (at the start of Section 3) will be taken to be sufficiently large. Given a finite set $S \subset \mathbb{N}$, write $\mathscr{P}(S)$ for the set of primes dividing some element of $S$, and $\mathscr{P}_{\text {small }}(S)$ for the set of primes $p \leqslant p_{0}$ dividing some element of $S$.

Our main result is the following.

Theorem 1.1. Let $X, Y, D \in[1, \infty)$, and suppose that $D \leqslant \min (X, Y)$. Let $\delta \in(0,1]$. Suppose that $A \subset[X, 2 X]$ and $B \subset[Y, 2 Y]$ are sets of integers with the following property: for at least $\delta|A||B|$ pairs $(a, b) \in A \times B, \operatorname{gcd}(a, b) \geqslant D$. Then we have the bound

$$
|A||B| \leqslant(1000)^{1+\# \mathscr{P}_{\text {small }}(A \cup B)} \delta^{-2-\varepsilon} \frac{X Y}{D^{2}} .
$$

Let us make some remarks on this theorem.

1. This obviously implies the cruder bound $|A||B| \ll \delta^{-2-\varepsilon} X Y / D^{2}$, mentioned in the abstract. The more precise form we have stated seems of little additional interest in its own right, but is critical for the proof. Perhaps the most natural case is when $A=B$ and $X=Y$, when the result says the following: if, for a proportion $\delta$ of all pairs $\left(a, a^{\prime}\right) \in A \times A$ we have $\operatorname{gcd}\left(a, a^{\prime}\right) \geqslant D$, then $|A| \ll \delta^{-1-\varepsilon} X / D$.

2. We believe that the result is new even when $\delta=1$, that is to say when $\operatorname{gcd}(a, b) \geqslant D$ for all $a \in A$ and $b \in B$. In this case, the result is clearly sharp up to a multiplicative constant. Indeed,

The first-named author is supported by a Simons Investigator Award and is grateful to the Simons Foundation for their support. The second-named author is supported by a Postdoctoral Fellowship with the Centre de Recherches Mathématiques and by a Junior Research Fellowship from Trinity College Cambridge. 
assuming that $D$ is an integer, we may take $A=\{x \in[X, 2 X]: D \mid x\}$ and $B=\{y \in[Y, 2 Y]: D \mid y\}$. One might wonder whether all the tight examples have approximately such a structure; however, Chow has constructed a different family of tight examples for $\delta=1$ (see [1, Section 15]) in which there is no single $d \gg D$ that divides a positive proportion of $A$ and $B$.

3. For $\delta \in(0,1)$ the result is also sharp for a wide range of parameters, apart from the factor of $\delta^{-\varepsilon}$. To see this, let $D \geqslant \delta^{-1}$ be given, set $D_{0}:=\lfloor\delta D\rfloor$, and consider the sets $A=B=$ $\left\{x \in[X, 2 X]: D_{0} \mid x\right\}$. Evidently, $|A||B| \sim \delta^{-2} X^{2} / D^{2}$. However, if $x=D_{0} m, x^{\prime}=D_{0} m^{\prime}$ with $m, m^{\prime} \in$ $\left[X / D_{0}, 2 X / D_{0}\right]$ and $\operatorname{gcd}\left(m, m^{\prime}\right) \geqslant D / D_{0}$, then $x, x^{\prime} \in A$ and $\operatorname{gcd}\left(x, x^{\prime}\right) \geqslant D$. The proportion of pairs of integers with gcd $k$ is $1 / k^{2} \zeta(2)$, and so the proportion of pairs of integers with gcd $\geqslant k$ is $\gg 1 / k$. It follows (at least if $X / D_{0}$ is big enough compared to $D / D_{0}$ ) that the number of such pairs $\left(x, x^{\prime}\right)$ is $\gg \delta|A||B|$.

4. When $A=B, X=Y$ and $\delta=1$, the result says the following: if $\operatorname{gcd}\left(a, a^{\prime}\right) \geqslant D$ for all $a, a^{\prime} \in A$, then $|A| \ll X / D$. However (we are rather embarrassed to admit), Zachary Chase pointed out to the authors that this particular result is trivial, because the assumption implies that $\left|a-a^{\prime}\right| \geqslant D$ whenever $a \neq a^{\prime}$. This argument does not, however, appear to extend to the other cases.

5. A straightforward dyadic decomposition argument would allow one to establish similar results under the assumption that $A \subset[X]$ and $B \subset[Y]$. We leave the details to the reader.

Notation. Our notation is standard. If $p$ is a prime and $a \in \mathbb{Z}$, we write $v_{p}(a)$ for the largest $k$ such that $p^{k} \mid a$. We extend this to rationals by $v_{p}(a / b)=v_{p}(a)-v_{p}(b)$. Implied constants in the $O(), \ll$ and $\gg$ notations are absolute (though they may depend on $\varepsilon$, which is fixed throughout the paper).

Strategy. Our strategy for proving Theorem 1.1 is essentially to proceed by induction on \# $\mathscr{P}(A \cup$ $B)$, but we will phrase the argument in terms of a hypothetical counterexample with minimal $\# \mathscr{P}(A \cup B)$. The first main business is to show that such a minimal counterexample has a very specific structure.

Proposition 1.2. Suppose we have a counterexample to Theorem 1.1 with the set $\mathscr{P}(A \cup B)$ minimal in size. Let $\Omega \subset A \times B$, with $|\Omega|=\delta|A||B|$, be the set of pairs for which $\operatorname{gcd}(a, b) \geqslant D$. Then there is $\Omega^{\prime} \subset \Omega$, with $\left|\Omega^{\prime}\right| \geqslant \frac{1}{2}|\Omega|$, and an integer $N$ such that the following is true. For all primes $p$ and for all $(a, b) \in \Omega^{\prime}$ we have $\left|v_{p}(a / N)\right|+\left|v_{p}(b / N)\right| \leqslant 1$.

Though such a statement does not appear explicitly in their work, this proposition should be considered essentially due to Koukoulopoulos and Maynard [1]. We will give a fairly short, selfcontained proof. On some level, this is equivalent to the argument of [1], but we phrase things rather differently.

To complete the proof of Theorem 1.1, we prove the following counterpart to Proposition 1.2.

Proposition 1.3. Suppose that $A \subset[X, 2 X], B \subset[Y, 2 Y], \Omega, D, \delta, N$ are as in Proposition 1.2. Then $|A||B| \leqslant 1000 \delta^{-2} X Y / D^{2}$.

Evidently, this means that $A, B$ do not in fact give a counterexample to Theorem 1.1. Combining Propositions 1.2 and 1.3 shows that no minimal counterexample to Theorem 1.1 exists, so Theorem 1.1 is true.

The proof of Proposition 1.3 uses some combinatorial arguments and is not found in [1].

\section{Concentrated measures on $\mathbb{Z}^{2}$}

In this section, we prove a result about concentration of probability measures on $\mathbb{Z}^{2}$. It is the key technical ingredient in the proof of Proposition 1.2, where it is used to concentrate the pair of valuation functions $\left(v_{p}(a), v_{p}(b)\right)$ around a diagonal pair $(k, k)$. 
Here, as in the rest of the paper, we write $q=2+\varepsilon$ and write $q^{\prime}$ for the conjugate index to $q$ (i.e. $\frac{1}{q}+\frac{1}{q^{\prime}}=1$ ).

Lemma 2.1. Let $c \leqslant 1$ and $\lambda \leqslant \frac{4}{5}$. Suppose that $\mu$ is a finitely supported probability measure on $\mathbb{Z}^{2}$. Suppose that there are sequences $x=\left(x_{i}\right)_{i \in \mathbb{Z}}, y=\left(y_{j}\right)_{j \in \mathbb{Z}}$ of non-negative reals such that $\|x\|_{\ell^{q^{\prime}}(\mathbb{Z})}=$ $\|y\|_{\ell q^{\prime}(\mathbb{Z})}=1$, and such that for all $(i, j) \in \mathbb{Z}^{2}$ we have

$$
\mu(i, j) \leqslant c \lambda^{|i-j|} x_{i} y_{j}
$$

Then $c \geqslant \frac{1}{9}$, and $\mu$ is highly concentrated near some point $(k, k)$ :

$$
\sum_{|i-k|+|j-k| \geqslant 2} \mu(i, j) \ll \lambda^{q+\varepsilon}
$$

Proof. We first prove the lower bound on $c$. Using (2.1), $\sum_{\mathbb{Z}^{2}} \mu(i, j)=1, \sum_{m \in \mathbb{Z}} \lambda^{|m|} \leqslant 9$ and $q^{\prime}<2$, we have

$$
\frac{1}{c} \leqslant \sum_{i, j} \lambda^{|i-j|} x_{i} y_{j} \leqslant \sum_{i, j} \lambda^{|i-j|}\left(x_{i} y_{j}\right)^{\frac{q^{\prime}}{2}} \leqslant 9 \sup _{m} \sum_{i}\left(x_{i} y_{i+m}\right)^{\frac{q^{\prime}}{2}} \leqslant 9,
$$

where the last step follows from the Cauchy-Schwarz inequality and the assumption $\|x\|_{\ell^{q^{\prime}}(\mathbb{Z})}=$ $\|y\|_{\ell q^{\prime}(\mathbb{Z})}=1$. The lower bound on $c$ follows.

Turning to (2.2), write $\sup _{i} x_{i} y_{i}=1-\gamma$ for some $\gamma \in[0,1]$, and suppose this supremum attained when $i=k$. Then, $x_{k}, y_{k} \geqslant 1-\gamma$, so

$$
\sum_{i \neq k} x_{i}^{q^{\prime}}, \sum_{j \neq k} y_{j}^{q^{\prime}} \ll \gamma \text { and } x_{i}, y_{j} \ll \gamma^{1 / q^{\prime}} \text { when } i, j \neq k \text {. }
$$

For $n=1,2,3,4,5,6$ write $\Sigma_{n}:=\sum_{(i, j) \in S_{n}} \mu(i, j)$, where $S_{1}, \ldots, S_{6}$ are the following sets, which partition $\mathbb{Z}^{2}$ :

$$
\begin{aligned}
& S_{1}:=\left\{(i, j) \in \mathbb{Z}^{2}: i \neq j \neq k \neq i\right\}, \quad S_{2}:=\{(k, j):|j-k| \geqslant 2\}, \\
& S_{3}:=\{(i, k):|i-k| \geqslant 2\}, \quad S_{4}:=\{(k, k \pm 1),(k \pm 1, k)\}, \quad S_{5}:=\{(i, i): i \neq k\},
\end{aligned}
$$

and finally $S_{6}:=\{(k, k)\}$. We bound the $\Sigma_{n}$ in turn.

Bound for $\Sigma_{1}$. By (2.1) and (2.3), we have

$$
\Sigma_{1} \leqslant \sum_{\substack{i, j \neq k \\ i \neq j}} \lambda^{|i-j|} x_{i} y_{j} \ll \gamma^{\frac{2}{q^{\prime}}\left(1-\frac{q^{\prime}}{2}\right)} \sum_{\substack{i, j \neq k \\ i \neq j}} \lambda^{|i-j|}\left(x_{i} y_{j}\right)^{\frac{q^{\prime}}{2}}=\gamma^{\frac{2}{q^{\prime}}-1} \sum_{m \neq 0} \lambda^{|m|} \sum_{\substack{i, i+m \neq k\\}}\left(x_{i} y_{i+m}\right)^{\frac{q^{\prime}}{2}} .
$$

By Cauchy-Schwarz and (2.3), for each fixed $m$ we have

$$
\sum_{i, i+m \neq k}\left(x_{i} y_{i+m}\right)^{\frac{q^{\prime}}{2}} \leqslant\left(\sum_{i \neq k} x_{i}^{q^{\prime}}\right)^{1 / 2}\left(\sum_{j \neq k} y_{j}^{q^{\prime}}\right)^{1 / 2} \ll \gamma
$$

Since $\sum_{m \neq 0} \lambda^{|m|} \ll \lambda$, putting these together gives $\Sigma_{1} \ll \lambda \gamma^{\frac{2}{q^{\prime}}}$. 
Bounds for $\Sigma_{2}, \Sigma_{3}$. For $\Sigma_{2}$, we use the trivial bound $x_{k} \leqslant 1$ and (2.3) for $y_{j}$. This gives (using the assumption that $\lambda \leqslant \frac{4}{5}$ )

$$
\Sigma_{2} \leqslant \sum_{|j-k| \geqslant 2}(5 \lambda / 4)^{|j-k|}(4 / 5)^{|j-k|} y_{j} \ll \lambda^{2} \sum_{|j-k| \geqslant 2}(4 / 5)^{|j-k|} y_{j} \ll \gamma^{\frac{1}{q^{\prime}}\left(1-\frac{q^{\prime}}{2}\right)} \lambda^{2} \sum_{|j-k| \geqslant 2}(4 / 5)^{|j-k|} y_{j}^{\frac{q^{\prime}}{2}} .
$$

By Cauchy-Schwarz and (2.3),

$$
\sum_{|j-k| \geqslant 2}(4 / 5)^{|j-k|} y_{j}^{q^{\prime}} \ll\left(\sum_{j \neq k}(4 / 5)^{2|j-k|}\right)^{1 / 2}\left(\sum_{j \neq k} y_{j}^{q^{\prime}}\right)^{1 / 2} \ll \gamma^{1 / 2}
$$

Combining these bounds gives $\Sigma_{2} \ll \gamma^{\frac{1}{q^{\prime}}} \lambda^{2}$, and an essentially identical argument yields $\Sigma_{3} \ll$ $\gamma^{\frac{1}{q^{\prime}}} \lambda^{2}$

Bound for $\Sigma_{4}$. From (2.1), (2.3) we immediately get $\Sigma_{4} \ll \gamma^{\frac{1}{q^{\prime}}} \lambda$.

Bound for $\Sigma_{5}$. A trivial modification to the argument used for $\Sigma_{1}$ (allowing $i=j$, which gives just a term with $m=0$ ) shows that $\Sigma_{5} \ll \gamma^{\frac{2}{q^{\prime}}}$.

Bound for $\Sigma_{5}+\Sigma_{6}$. By (2.1) and the fact that $\sup _{i} x_{i} y_{i}=1-\gamma$,

$$
\Sigma_{5}+\Sigma_{6} \leqslant \sum_{i} x_{i} y_{i} \leqslant(1-\gamma)^{1-\frac{q^{\prime}}{2}} \sum_{i}\left(x_{i} y_{i}\right)^{\frac{q^{\prime}}{2}} \leqslant(1-\gamma)^{1-\frac{q^{\prime}}{2}} \leqslant 1-\left(1-\frac{q^{\prime}}{2}\right) \gamma,
$$

where we used Cauchy-Schwarz yet again.

Putting all this together gives

$$
1=\sum_{n=1}^{6} \Sigma_{n} \leqslant 1-\left(1-\frac{q^{\prime}}{2}\right) \gamma+O\left(\lambda \gamma^{\frac{1}{q^{\prime}}}\right)
$$

This implies that $\lambda \gg \gamma^{1-\frac{1}{q^{\prime}}}$, i.e. $\gamma \ll \lambda^{q}$. Finally, we see that

$$
\sum_{|i-k|+|j-k| \geqslant 2} \mu(i, j)=\Sigma_{1}+\Sigma_{2}+\Sigma_{3}+\Sigma_{5} \ll \lambda^{\frac{2 q}{q^{\prime}}}+\lambda^{2+\frac{q}{q^{\prime}}} \ll \lambda^{\frac{2 q}{q^{\prime}}}
$$

(since $q \leqslant 3$ ). The result follows, noting that $\frac{2 q}{q^{\prime}}=q+\varepsilon$.

\section{Properties of a minimal counterexample}

We turn now to the proof of Proposition 1.2. We first reduce matters to the following "local" statement at a single prime $p$.

Proposition 3.1. Suppose we have a counterexample to Theorem 1.1 with the set $\mathscr{P}(A, B)$ minimal in size. Let $\Omega \subset A \times B$, with $|\Omega|=\delta|A||B|$, be the set of pairs for which $\operatorname{gcd}(a, b) \geqslant D$. Let $p \in \mathscr{P}(A, B)$ be a prime. Then, $p>p_{0}(\varepsilon)$, and there is $k_{p} \in \mathbb{Z}_{\geqslant 0}$ and $\Omega_{p} \subset \Omega$ such that for all $(a, b) \in \Omega_{p}$ we have $\left|v_{p}(a)-k_{p}\right|+\left|v_{p}(b)-k_{p}\right| \leqslant 1$, and such that $\left|\Omega \backslash \Omega_{p}\right| \ll p^{-1-\varepsilon / 3}|\Omega|$. 
Proposition 1.2 follows quickly from this by taking $N=\prod_{p} p^{k_{p}}$ and $\Omega^{\prime}:=\bigcap_{p} \Omega_{p}$. We have

$$
\left|\Omega^{\prime}\right| \geqslant|\Omega|\left(1-O\left(\sum_{p>p_{0}} p^{-1-\varepsilon / 3}\right)\right) \geqslant \frac{1}{2}|\Omega|,
$$

if $p_{0}$ is big enough (this is the point at which $p_{0}$ is constrained).

It remains, then, to establish Proposition 3.1. Fix, for the rest of this section, the prime $p$. For $i, j \in \mathbb{Z}_{\geqslant 0}$, we define $A_{i}:=\left\{a \in A: v_{p}(a)=i\right\}, B_{j}:=\left\{b \in B: v_{p}(b)=j\right\}$, and write $\alpha_{i}:=\frac{\left|A_{i}\right|}{|A|}$ and $\beta_{j}:=\frac{\left|B_{j}\right|}{|B|}$ for the relative densities of these sets. Write $\mu(i, j):=\frac{\left|\Omega \cap\left(A_{i} \times B_{j}\right)\right|}{|\Omega|}$, thus $\mu$ is a finitely supported probability measure on $\mathbb{Z}_{2}^{2}$.

For any $i, j$, consider the sets $\bar{A}_{i}:=p^{-i} \cdot A_{i}$ and $\bar{B}_{j}:=p^{-j} \cdot B_{j}$. These are sets of integers, coprime to $p$, with $\bar{A}_{i} \subset\left[\frac{X}{p^{i}}, \frac{2 X}{p^{i}}\right], \bar{B}_{j} \subset\left[\frac{Y}{p^{j}}, \frac{2 Y}{p^{j}}\right]$ and $\operatorname{gcd}(x, y) \geqslant \frac{D}{p^{\min (i, j)}}$ whenever $x=p^{-i} a, y=p^{-j} b$ with $(a, b) \in \Omega$.

By the minimality assumption, these sets cannot be a counterexample to Theorem 1.1, and therefore we have the inequality

$$
\left|\bar{A}_{i}\right|\left|\bar{B}_{j}\right| \leqslant(1000)^{1+\# \mathscr{P}_{\text {small }}\left(\bar{A}_{i}, \bar{B}_{j}\right)}\left(\frac{\delta \mu(i, j)}{\alpha_{i} \beta_{j}}\right)^{-2-\varepsilon} \frac{\frac{X}{p^{i}} \frac{Y}{p^{j}}}{\left(\frac{D}{p^{\min (i, j)}}\right)^{2}} .
$$

On the other hand,

$$
\left|\bar{A}_{i}\right|\left|\bar{B}_{j}\right|=\alpha_{i} \beta_{j}|A||B| \geqslant(1000)^{1+\# \mathscr{P}_{\text {small }}(A, B)} \alpha_{i} \beta_{j} \delta^{-2-\varepsilon} \frac{X Y}{D^{2}} .
$$

Note also that $\mathscr{P}\left(\bar{A}_{i}, \bar{B}_{j}\right) \subset \mathscr{P}(A, B) \backslash\{p\}$, and so

$$
\# \mathscr{P}_{\text {small }}\left(\bar{A}_{i}, \bar{B}_{j}\right) \leqslant \# \mathscr{P}_{\text {small }}(A, B)-1_{p \leqslant p_{0}} .
$$

Comparing (3.1), (3.2), and (3.3) gives, for all $i$ and $j$,

$$
\mu(i, j) \leqslant 10^{-1_{p} \leqslant p_{0}}\left(\alpha_{i} \beta_{j}\right)^{\frac{1+\varepsilon}{2+\varepsilon}} p^{-\frac{|i-j|}{2+\varepsilon}},
$$

since $\varepsilon<1$. This puts us in the situation covered by Lemma 2.1, with (in that lemma)

$$
q=2+\varepsilon, q^{\prime}=\frac{2+\varepsilon}{1+\varepsilon}, \lambda=p^{-\frac{1}{q}}, c=\left(\frac{1}{10}\right)^{1_{p \leqslant p_{0}}}, x_{i}:=\alpha_{i}^{1 / q^{\prime}}, y_{j}:=\beta_{j}^{1 / q^{\prime}} .
$$

The hypotheses of the lemma are satisfied, since $p^{-\frac{1}{q}} \leqslant 2^{-\frac{1}{3}} \leqslant 4 / 5$. The lemma implies, first of all, that $c>\frac{1}{10}$; this immediately tells us that $p>p_{0}$. We conclude that there is some $k$ such that

$$
\sum_{|i-k|+|j-k| \geqslant 2} \mu(i, j) \ll \lambda^{q+\varepsilon} \ll p^{-1-\varepsilon / 3} .
$$

This is precisely what is needed in Proposition 3.1, taking

$$
\Omega_{p}=\bigcup_{|i-k|+|j-k| \leqslant 1}\left(\Omega \cap\left(A_{i} \times B_{j}\right)\right) .
$$

\section{Finishing the argument}

In this section, we complete the proof of Theorem 1.1 by establishing Proposition 1.3. That is, our task is as follows. Suppose that $A \subset[X, 2 X], B \subset[Y, 2 Y]$, that $\Omega \subset A \times B$ has size $\frac{\delta}{2}|A||B|$, and that $\operatorname{gcd}(a, b) \geqslant D$ whenever $(a, b) \in \Omega$. Suppose that there is some positive integer $N$ such that 


$$
\left|v_{p}(a / N)\right|+\left|v_{p}(b / N)\right| \leqslant 1,
$$

for all primes $p$ and for all $(a, b) \in \Omega$. We are to show that, under these assumptions, we have the bound

$$
|A||B| \leqslant \frac{1000}{\delta^{2}} \frac{X Y}{D^{2}} .
$$

Let us begin the proof. In the course of the argument, it will be convenient to use a little of the language of graph theory. Thus, if $a \in A$, then we write $\operatorname{deg}(a):=\#\{b \in B:(a, b) \in \Omega\}$, and analogously for $b \in B$. Write $A^{\prime}:=\{a \in A: \operatorname{deg}(a)>0\}$ and $B^{\prime}:=\{b \in B: \operatorname{deg}(b)>0\}$.

If $a \in A^{\prime}$ then, by (4.1), $v_{p}(a / N) \in\{-1,0,1\}$ for all primes $p$. We define the defect $a_{*}$ to be the product of all primes for which $v_{p}(a / N) \neq 0$. Now we make the crucial observation that if $(a, b) \in \Omega$ then

$$
a_{*} b_{*}=\frac{a b}{\operatorname{gcd}(a, b)^{2}} .
$$

To prove this, we take $p$-adic valuations. It is easily seen that

$$
v_{p}\left(a b / \operatorname{gcd}(a, b)^{2}\right)=\left|v_{p}(a)-v_{p}(b)\right|=\left|v_{p}(a / N)-v_{p}(b / N)\right|
$$

so we need only prove that

$$
v_{p}\left(a_{*}\right)+v_{p}\left(b_{*}\right)=\left|v_{p}(a / N)-v_{p}(b / N)\right|,
$$

whenever $(a, b) \in \Omega$. This follows immediately from (4.1), noting that $v_{p}\left(a_{*}\right)=1$ if $v_{p}(a / N)= \pm 1$ and $v_{p}\left(a_{*}\right)=0$ otherwise, and similarly for $v_{p}\left(b_{*}\right)$.

As a consequence of (4.3) and our assumptions, we see that

$$
a_{*} b_{*} \leqslant \frac{4 X Y}{D^{2}},
$$

whenever $(a, b) \in \Omega$. This would allow us to conclude very quickly, were it not for the fact that the map $a \mapsto a_{*}$ need not be injective (see Section 5 for some further remarks on this point). Fortunately, we have the following substitute for injectivity.

Lemma 4.1. Let $T \in \mathbb{R}_{>0}$. The number of $a \in A^{\prime}$ for which $a_{*} \leqslant T$ is at most $2 T$. Similarly, the number of $b \in B^{\prime}$ for which $b_{*} \leqslant T$ is at most $2 T$.

Proof. If $a \in A^{\prime}$, write $a_{+}$for the product of all primes with $v_{p}(a / N)=1$, and $a_{-}$for the product of all primes with $v_{p}(a / N)=-1$. Thus,

$$
a_{*}=a_{+} a_{-}
$$

Since $v_{p}(a / N) \in\{-1,0,1\}$, we have

$$
\frac{a_{+}}{a_{-}}=\frac{a}{N}
$$

Since $A \subset[X, 2 X]$, it follows by multiplying (4.6) and (4.7) that if $a_{*} \leqslant T$ then

$$
a_{+} \leqslant\left(\frac{a T}{N}\right)^{1 / 2} \leqslant\left(\frac{2 X T}{N}\right)^{1 / 2}
$$


Similarly, dividing (4.6) by (4.7), we see that if $a_{*} \leqslant T$ then

$$
a_{-} \leqslant\left(\frac{N T}{a}\right)^{1 / 2} \leqslant\left(\frac{N T}{X}\right)^{1 / 2} .
$$

It follows from (4.8), (4.9) that the number of choices for the pair $\left(a_{+}, a_{-}\right)$is at most $2 \mathrm{~T}$. However, if we know $a_{+}, a_{-}$and $N$ then we can recover $a$ uniquely, so the map $a \mapsto\left(a_{+}, a_{-}\right)$is injective. The proof for $B^{\prime}$ is the same.

Now we finish the argument. By a standard averaging argument there is a set $\tilde{A} \subset A$ with $|\tilde{A}| \geqslant$ $\delta|A| / 4$ such that $\operatorname{deg}(a) \geqslant \delta|B| / 4$ for all $a \in \tilde{A}$. Clearly, $\tilde{A} \subset A^{\prime}$, so by Lemma 4.1 there is some $a \in \tilde{A}$ such that

$$
a_{*} \geqslant \delta|A| / 8 .
$$

Set $\tilde{B}:=\{b \in B:(a, b) \in \Omega\}$. Thus, $|\tilde{B}| \geqslant \delta|B| / 4$. Clearly $\tilde{B} \subset B^{\prime}$, so by Lemma 4.1 there is some $b \in \tilde{B}$ such that

$$
b_{*} \geqslant \delta|B| / 8 .
$$

By construction we have $(a, b) \in \Omega$, so we have the upper bound (4.5).

Comparing (4.5), (4.10), (4.11) immediately yields (4.2).

\section{Further results and remarks}

Suppose that $A$ and $B$ are finite sets of square-free positive integers. In this instance, we may assume that the positive integer $N$ that satisfies (4.1) is also square-free, and thus the map $a \mapsto a_{*}$ is injective, since knowing $N$ and $a_{*}$ determines $a$. This enables us to circumvent Lemma 4.1 , and prove the following theorem.

Theorem 5.1. Let $Q \in[1, \infty)$ and $\delta \in(0,1]$. Suppose that $A, B$ are finite sets of square-free positive integers with the following property: for at least $\delta|A||B|$ pairs $(a, b) \in A \times B, a b / \operatorname{gcd}(a, b)^{2} \leqslant Q$. Then we have the bound

$$
|A||B| \leqslant(1000)^{1+\# \mathscr{P}_{\text {small }}(A \cup B)} \delta^{-2-\varepsilon} \frac{Q}{4} .
$$

Of course this result implies the cruder bound $|A||B| \ll \delta^{-2-\varepsilon} Q$. It also implies Theorem 1.1, upon taking $Q=4 X Y / D^{2}$.

Proof. The proof of Proposition 1.2 holds mutatis mutandis. Analysing the minimal counterexample as before, we conclude that $a_{*} b_{*} \leqslant Q$ (by analogy with (4.5)). Using graph theoretic language as before, there exists a set $\tilde{A} \subset A$ with $|\tilde{A}| \geqslant \delta|A| / 4$ such that $\operatorname{deg}(a) \geqslant \delta|B| / 4$ for all $a \in \tilde{A}$. Since $a \mapsto a_{*}$ is injective, there is some $a \in \tilde{A}$ for which $a_{*} \geqslant \delta|A| / 4$. Letting $\tilde{B}:=\{b \in$ $B:(a, b) \in \Omega\}$, we have $|\tilde{B}| \geqslant \delta|B| / 4$ and for all $b \in \tilde{B}$ we have $b_{*} \leqslant Q / a_{*}$. Therefore, since $b \mapsto b_{*}$ is injective, we have

$$
\frac{\delta|B|}{4} \leqslant|\tilde{B}| \leqslant \max _{b_{*}: b \in \tilde{B}} b^{*} \leqslant \frac{Q}{a_{*}} \leqslant \frac{4 Q}{\delta|A|} .
$$

This rearranges to $|A||B| \leqslant 16 \delta^{-2} Q$, which shows that the minimal counterexample is not in fact a counterexample, thus settling the theorem.

One might wonder whether the bound $|A||B| \ll \delta^{-2-\varepsilon} Q$ holds for general finite sets of integers $A$ and $B$ (not just for square-frees). However, there is a counterexample to this assertion, even with $\delta=1$, given by 


$$
A=B=\left\{\left(\prod_{p \leqslant X} p\right) \frac{m}{n}: m n \leqslant X, \mu^{2}(m)=\mu^{2}(n)=1, \operatorname{gcd}(m, n)=1\right\}
$$

One may establish that for all $\left(a_{1}, a_{2}\right) \in A \times A$ one has the bound $a_{1} a_{2} / \operatorname{gcd}\left(a_{1}, a_{2}\right)^{2} \leqslant X^{2}$. Yet $|A| \gg X \log X$.

By this, one notes that the use of dyadic ranges in the proof of Lemma 4.1 was critical.

\section{Acknowledgements}

We would like to thank Andrew Granville, Dimitris Koukoulopoulos, and James Maynard for interesting conversations related to this work.

\section{Reference}

[1] Koukoulopoulos, D. and Maynard, J. (2020) On the Duffin-Schaeffer conjecture. Ann. Math. 192(1) 251-307. 